\title{
Security of Blind Signatures under Aborts
}

\author{
Marc Fischlin and Dominique Schröder \\ Darmstadt University of Technology, Germany \\ marc.fischlin@gmail.com, schroeder@me.com, \\ www.minicrypt.de
}

\begin{abstract}
We explore the security of blind signatures under aborts where the user or the signer may stop the interactive signature issue protocol prematurely. Several works on blind signatures discuss security only in regard of completed executions and usually do not impose strong security requirements in case of aborts. One of the exceptions is the paper of Camenisch, Neven and shelat (Eurocrypt 2007) where the notion of selective-failure blindness has been introduced. Roughly speaking, selective-failure blindness says that blindness should also hold in case the signer is able to learn that some executions have aborted.

Here we augment the work of Camenisch et al. by showing how to turn every secure blind signature scheme into a selective-failure blind signature scheme. Our transformation only requires an additional computation of a commitment and therefore adds only a negligible overhead. We also study the case of multiple executions and notions of selectivefailure blindness in this setting. We then discuss the case of user aborts and unforgeability under such aborts. We show that every three-move blind signature scheme remains unforgeable under such user aborts. Together with our transformation for selective-failure blindness we thus obtain an easy solution to ensure security under aborts of either party and which is applicable for example to the schemes of Pointcheval and Stern (Journal of Cryptology, 2000).

We finally revisit the construction of Camenisch et al. for simulatable adaptive oblivious transfer protocols, starting from selective-failure blind signatures where each message only has one valid signature (uniqueness). While our transformation to achieve selective-failure blindness does not preserve uniqueness, it can still be combined with a modified version of their protocol. Hence, we can derive such oblivious transfer protocols based on unique blind signature schemes only (in the random oracle model), without necessarily requiring selective-failure blindness from scratch.
\end{abstract}

\section{Introduction}

Blind signatures, proposed by Chaum [5], allow a signer to interactively sign messages for users such that the messages are hidden from the signer. Since their introduction many blind signatures schemes have been proposed 1 : $35: 0,9: 10: 17$, $18,21,22]$, and they typically share two basic security properties: blindness says that a malicious signer cannot decide upon the order in which two messages have

The original version of this chapter was revised: The copyright line was incorrect. This has been corrected. The Erratum to this chapter is available at DOI: 10.1007/978-3-642-00468-1_29

S. Jarecki and G. Tsudik (Eds.): PKC 2009, LNCS 5443, pp. 297-316, 2009.

(C) Springer-Verlag Berlin Heidelberg 2009 
been signed in two executions with an honest user, and unforgeability demands that no adversarial user can create more signatures than interactions with the honest signer took place.

The security requirements for blind signatures have been formalized by Juels et al. [16] and by Pointcheval and Stern 22. Although these widely used definitions give basic security guarantees, blindness only holds in a restricted sense when it comes to aborted executions. That is, prior work does not guarantee blindness in case the signer is able to learn which of two executions aborted (even if one execution aborts only after the protocol has concluded)". However, in e-cash scenarios an honest user, unable to eventually derive a valid coin, will most likely complain to the malicious bank afterwards.

Recently, Camenisch et al. 7] consider a stronger kind of aborts where a cheating signer may be able to make the user algorithm fail depending on the message being signed 1 and where the malicious signer is informed afterwards which execution has failed (if any). Considering for example a voting protocol based on blind signatures 7 10, a malicious administrator can potentially deduce information about votes (possibly also for non-aborted executions) by causing some voters to abort and consulting the subsequent complaints.

As for user aborts and unforgeability, albeit the definitions $[16$ and 22 are identical in spirit, the "one-more" notion in [22] leaves two possible interpretations: either the adversarial user is deemed to generate one more signature than executions with the signer have been initiated (i.e., even counting executions in which the user aborts), or the malicious user needs to output one more signature than executions have been completed (i.e., allowing user aborts). In fact, this ambiguity re-appears in many works about blind signatures, some explicitly counting initiated executions [3, 9, 15, some emphatically referring to completed executions [6, 16, 18, 21] and some remaining vague, too [1,7,14.

For both cases, user and signer aborts, the stronger notions are desirable of course. For a blind signature scheme used to sign coins in an e-cash system, for instance, a malicious signer may otherwise abort executions deliberately and, by this, may be able to revoke unlinkability of coins. Vice versa, if unforgeability says that no adversarial user is able to create more signatures than interactions with the signer have been initiated, and no requirement about aborted sessions is imposed, then an adversarial user could potentially derive more signatures from such aborted executions. The signing bank could generally charge users for executions, which have stopped early. Yet, if the connection in the signing process breaks down accidently, the honest user is most likely unable to derive the coin and would hence be reluctant to pay for the transaction The bank may then gracefully waive the fee for such aborted executions, but still needs to handle forgery attempts.

Related Work. As mentioned before, Camenisch et al. 7. have already considered the limitations of the standard blindness notion. They have introduced an extension called selective-failure blindness in which the a malicious signer

${ }^{1}$ Ultimately, since the malicious signer causes the abort, this can be seen as a more general case of signer aborts. 
should not be able to force an honest user to abort the signature issue protocol because of a certain property of the user's message, which would disclose some information about the message to the signer. They present a construction of a simulatable oblivious transfer protocols from so-called unique selective-failure blind signature schemes (in the random oracle model) for which the signature is uniquely determined by the message. Since the main result of the work 7 is the construction of oblivious transfer protocols, the authors note that Chaum's scheme [5] and Boldyreva's protocol 3] are examples of such selective-failure blind schemes, but do not fully explore the relationship to (regular) blindness.

Hazay et al. 15 present a concurrently-secure blind signature scheme and, as part of this, they also introduce a notion called a-posteriori blindness. This notion considers blindness of multiple executions between the signer and the user (as opposed to two sessions as in the basic case), and addresses the question how to deal with executions in which the user cannot derive a signature. However, the definition of a-posteriori blindness is neither known to be implied by ordinary blindness, nor implies it ordinary blindness (as sketched in [15). Thus, selectivefailure blindness does not follow from this notion.

Aborts of players have also been studied under the notion of fairness in twoparty and multi-party computations, especially for the exchange of signatures, e.g. 2, 11, 13]. Fairness should guarantee that one party obtains the output of the joint computation if and only if the other party receives it. Note, however, that in case of blind signatures the protocol only provides a one-sided output to the user (namely, the signature). In addition, solutions providing fairness usually require extra assumptions like a trusted third party in case of disputes, or they add a significant overhead to the underlying protocol.

Our Results. We pick up the idea of selective-failure blindness to deal with signer aborts and expand the work of Camenisch et al. [7] towards its relationship to blindness and further constructions of such schemes. We first show that selective-failure blindness is indeed a strictly stronger notion than regular blindness. We also extend the notion of selective-failure blindness to multiple executions, particularly addressing aborts of a subset of executions. We give two possible definitions for the multi-execution case and prove them to be equivalent. We then show that blindness in the basic case of two executions suffices to guarantee security in the case of many sessions and discuss the relation to a-posteriori blindness [15].

Next we present a general transformation which turns every secure blind signature scheme into a selective-failure blind scheme. Our transformation only requires an additional commitment of the message, which the user computes before the actual protocol starts and which the user then uses in the original protocol instead of the message itself 2 Since the commitment is non-interactive, our transformation inherits important characteristics of the underlying protocol like the number of moves and concurrent security.

\footnotetext{
${ }^{2}$ This idea has been conjectured by Hazay et al. 15 to also work for a-posteriori blindness. We are not aware of any formal claim or proof in the literature that using a commitment indeed provides security against aborts.
} 
It should be noted, though, that the transformation destroys uniqueness (i.e., that each message has only one valid signature per key pair), as as required by [7] to derive oblivious transfer from such blind signatures. However, we show that our transformation is still applicable if we modify the oblivious transfer protocol of 7 slightly. Hence, we can now easily obtain an adaptive oblivious transfer from any unique blind signature scheme such that the protocol is simulatable in presence of failures. Put differently, we show that selective-failure blindness is not necessary to obtain such oblivious transfer protocols, but uniqueness is sufficient. We note that like the original protocol in [7] this result is in the random oracle model.

We finally study the case of user aborts and show that every three-move blind signature scheme is unforgeable under user aborts. While this is clear for twomove schemes like Chaum's protocol [5] our result shows that this remains true for other schemes like the ones by Pointcheval and Stern [22]. We show that, in general, this does not hold for schemes with four or more moves, assuming the existence of a secure two-move blind signature scheme. It remains open if there is a non-trivial and efficient transformation to take care of user aborts for schemes with more than three moves 3

In summary, our transformation to achieve selective-failure blindness, together with the result about user aborts, shows that any scheme with two or three moves can be efficiently turned into one, which is secure under aborts (of either party).

\section{Blind Signatures}

To define blind signatures formally we introduce the following notation for interactive executions between algorithms $\mathcal{X}$ and $\mathcal{Y}$. By $(a, b) \leftarrow\langle\mathcal{X}(x), \mathcal{Y}(y)\rangle$ we denote the joint execution of $\mathcal{X}$ and $\mathcal{Y}$, where $x$ is the private input of $\mathcal{X}$ and $y$ defines the private input of $\mathcal{Y}$. The private output of $\mathcal{X}$ equals $a$ and the private output of $\mathcal{Y}$ is $b$. We write $\mathcal{Y}^{\langle\mathcal{X}(x), \cdot\rangle^{\infty}}(y)$ if $\mathcal{Y}$ can invoke an unbounded number of executions of the interactive protocol with $\mathcal{X}$ in arbitrarily interleaved order. Accordingly, $\mathcal{X}\left\langle\cdot, \mathcal{Y}\left(y_{0}\right)\right\rangle^{1},\left\langle\cdot \mathcal{Y}\left(y_{1}\right)\right\rangle^{1}(x)$ can invoke arbitrarily ordered executions with $\mathcal{Y}\left(y_{0}\right)$ and $\mathcal{Y}\left(y_{1}\right)$, but interact with each algorithm only once.

Definition 1 (Blind Signature Scheme). A blind signature scheme consists of a tuple of efficient algorithms $\mathrm{BS}=\left(\mathrm{KG}_{\mathrm{BS}},\langle\mathcal{S}, \mathcal{U}\rangle, \mathrm{Vf}_{\mathrm{BS}}\right)$ where

Key Generation. $\mathrm{KG}_{\mathrm{BS}}\left(1^{n}\right)$ for parameter $n$ generates a key pair $\left(s k_{\mathrm{BS}}, p k_{\mathrm{BS}}\right)$.

Signature Issuing. The joint execution of algorithm $\mathcal{S}\left(\right.$ sk $\left._{\mathrm{BS}}\right)$ and algorithm $\mathcal{U}\left(p k_{\mathrm{BS}}, m\right)$ for message $m \in\{0,1\}^{n}$ generates an output $\sigma$ of the user (and some possibly empty output $\lambda$ for the signer $),(\lambda, \sigma) \leftarrow\left\langle\mathcal{S}\left(s k_{\mathrm{BS}}\right), \mathcal{U}\left(p k_{\mathrm{BS}}, m\right)\right\rangle$.

Verification. $\mathrm{Vf}_{\mathrm{BS}}\left(p k_{\mathrm{BS}}, m, \sigma\right)$ outputs a bit.

${ }^{3}$ By trivial transformations we refer for instance to a solution which ignores the underlying scheme and simply runs, say, Chaum's protocol. 
It is assumed that the scheme is complete, i.e., for any $n \in \mathbb{N}$, any $\left(s k_{\mathrm{BS}}, p k_{\mathrm{BS}}\right) \leftarrow$ $\mathrm{KG}_{\mathrm{BS}}\left(1^{n}\right)$, any message $m \in\{0,1\}^{n}$ and any $\sigma$ output by $\mathcal{U}$ in the joint execution of $\mathcal{S}\left(s k_{\mathrm{BS}}\right)$ and $\mathcal{U}\left(p k_{\mathrm{BS}}, m\right)$ we have $\mathrm{Vf}_{\mathrm{BS}}\left(p k_{\mathrm{BS}}, m, \sigma\right)=1$.

Security of blind signature schemes is defined by unforgeability and blindness 16, 22. An adversary $\mathcal{U}^{*}$ against unforgeability tries to generate $k+1$ valid message-signatures pairs after at most $k$ completed interactions with the honest signer, where the number of executions is adaptively determined by $\mathcal{U}^{*}$ during the attack. To identify completed sessions we assume that the honest signer returns a special symbol ok when having sent the final protocol message in order to indicate a completed execution (from its point of view). We remark that this output is "atomically" connected to the final transmission to the user.

The blindness condition says that it should be infeasible for a malicious signer $\mathcal{S}^{*}$ to decide which of two messages $m_{0}$ and $m_{1}$ has been signed first in two executions with an honest user $\mathcal{U}$. If one of these executions has returned $\perp$ then the signer is not informed about the other signature either.

Definition 2 (Secure Blind Signature Scheme). A blind signature scheme $\mathrm{BS}=\left(\mathrm{KG}_{\mathrm{BS}},\langle\mathcal{S}, \mathcal{U}\rangle, \mathrm{Vf}_{\mathrm{BS}}\right)$ is called secure if the following holds:

Unforgeability. For any efficient algorithm $\mathcal{U}^{*}$ the probability that experiment Unforge $_{\mathcal{U}^{*}}^{\mathrm{BS}}(n)$ evaluates to 1 is negligible (as a function of $n$ ) where

Experiment Unforge $\mathcal{U}^{\mathrm{BS}}(n)$

$$
\begin{aligned}
& \left(s k_{\mathrm{BS}}, p k_{\mathrm{BS}}\right) \leftarrow \mathrm{KG}_{\mathrm{BS}}\left(1^{n}\right) \\
& \left(\left(m_{1}, \sigma_{1}\right), \ldots,\left(m_{k+1}, \sigma_{k+1}\right)\right) \leftarrow \mathcal{U}^{*\left\langle\mathcal{S}\left(s k_{\mathrm{BS}}\right), \cdot\right\rangle^{\infty}}\left(p k_{\mathrm{BS}}\right) \\
& \text { Return } 1 \text { iff } \\
& \quad m_{i} \neq m_{j} \text { for } 1 \leq i<j \leq k+1 \text {, and } \\
& \quad \mathrm{Vf}_{\mathrm{BS}}\left(p k_{\mathrm{BS}}, m_{i}, \sigma_{i}\right)=1 \text { for all } i=1,2, \ldots, k+1, \text { and } \\
& \quad \mathcal{S} \text { has returned ok in at most } k \text { interactions. }
\end{aligned}
$$

Blindness. For any efficient algorithm $\mathcal{S}^{*}$ (working in modes find, issue and guess) the probability that the following experiment $\operatorname{Blind}_{\mathcal{S}^{*}}^{\mathrm{BS}}(n)$ evaluates to 1 is negligibly close to $1 / 2$, where

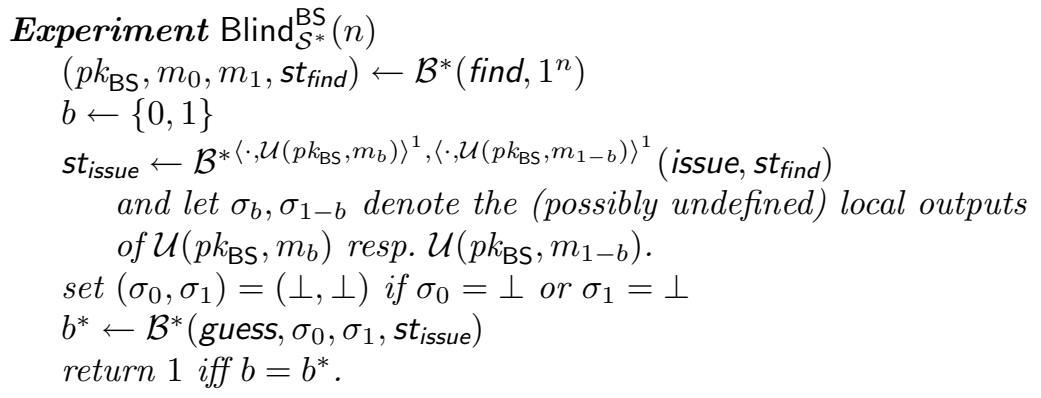

\section{Selective-Failure Blindness}

In this section we review the definition of selective-failure blindness and show that selective-failure blindness is a strictly stronger requirement than the basic 
blindness property. Second, we discuss how to extend selective-failure blindness to multiple executions.

\subsection{Definition}

Camenisch et al. 7] put forward the notion of selective-failure blindness, which says that a malicious signer $\mathcal{S}^{*}$ cannot force the user algorithm $\mathcal{U}$ to abort based on the specific message. This is formalized by informing $\mathcal{S}^{*}$ which instance has aborted (i.e., if the left, the right, or both user instances have failed):

Definition 3. A blind signature scheme $\mathrm{BS}=\left(\mathrm{KG}_{\mathrm{BS}},\langle\mathcal{S}, \mathcal{U}\rangle, \mathrm{Vf}_{\mathrm{BS}}\right)$ is called selective-failure blind if it is unforgeable (as in Definition [) and the following holds:

Selective-Failure Blindness. For any efficient algorithm $\mathcal{S}^{*}$ (which works in modes find, issue and guess) the probability that experiment $\operatorname{SFBlind}_{\mathcal{S}^{*}}^{\mathrm{BS}}(n)$ evaluates to 1 is negligibly close to $1 / 2$ where

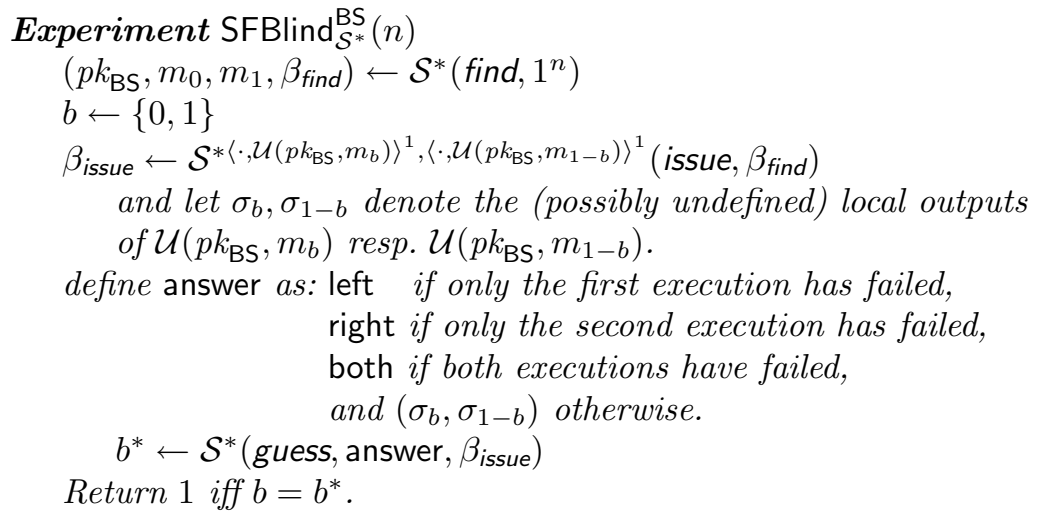

\subsection{Relation to Regular Blindness}

We first prove formally the fact that selective-failure blindness implies regular blindness. Then we separate the notion by turning a secure blind signature scheme into a one which is still secure but provably not selective-failure blind.

Proposition 1. Every selective-failure blind signature scheme $\mathrm{BS}_{\mathrm{SF}}$ is also a secure blind signature scheme.

The claim follows easily and the formal proof is given in the full version.

Proposition 2. If there exists a secure blind signature scheme BS, then there exists a secure blind signature scheme $\mathrm{BS}_{\overline{\mathrm{SF}}}$ which is not selective-failure blind.

Proof. We modify $\mathrm{BS}$ slightly into a scheme $\mathrm{BS}_{\overline{\mathrm{SF}}}$ which is identical to $\mathrm{BS}$, except that we modify the key generation algorithm and add a break condition into the user algorithm. More precisely, let $\mathrm{BS}=\left(\mathrm{KG}_{\mathrm{BS}},\langle\mathcal{S}, \mathcal{U}\rangle, \mathrm{Vf}_{\mathrm{BS}}\right)$ be a secure blind signature scheme. We define the new blind signature scheme $\mathrm{BS}_{\overline{\mathrm{SF}}}$ as 
KeyGen. $\mathrm{KG}_{\overline{\mathrm{SF}}}$ first sets $m_{\max }=1^{n}$ as the maximum of the lexicographical order over $n$-bit strings. It then executes the key generation algorithm of the underlying blind signature scheme $\left(s k_{\mathrm{BS}}, p k_{\mathrm{BS}}\right) \leftarrow \mathrm{KG}_{\mathrm{BS}}\left(1^{n}\right)$ and returns $\left(s k_{\overline{\mathrm{SF}}}, p k_{\overline{\mathrm{SF}}}\right)=\left(s k_{\mathrm{BS}},\left(p k_{\mathrm{BS}}, m_{\mathrm{max}}\right)\right)$.

Signing Protocol. The interactive signing protocol remains unchanged except for one modification. The user algorithm checks after the last move of the protocol (and after computing the signature $\sigma$ ) that $m \leq m_{\max }$ and, if so, returns the signature $\sigma$, and $\perp$ otherwise.

Verification. The verification algorithm returns the result of $\mathrm{Vf}_{\mathrm{BS}}$.

The modified scheme is clearly complete, as the case $m>m_{\max }$ for an honest signer never occurs and because the initial protocol is complete. Obviously, if the blind signature scheme $\mathrm{BS}$ is unforgeable, then $\mathrm{BS}_{\overline{\mathrm{SF}}}$ is also unforgeable. This is easy to see as the malicious user may simply ignore the break condition.

Concerning blindness, first note that the malicious signer $\mathcal{S}^{*}$ is allowed to choose the public key and thus to pick some other value $m_{\max }^{*}$. As a malicious signer $\mathcal{S}^{*}$ is not informed which of the executions has failed (if any), setting some other value $m_{\max }^{*}$ than the predetermined maximum and possibly causing an abort does not lend any additional power to $\mathcal{S}^{*}$. To see this, note that the user algorithm does not abort prematurely if $m>m_{\max }$. Hence, from the (malicious) signer's point of view, the interaction is indistinguishable from an honest execution. It therefore follows that $\mathrm{BS}_{\overline{\mathrm{SF}}}$ still satisfies blindness.

We finally show that the modified scheme does not fulfill selective-failure

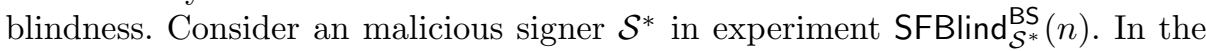
first step the adversary $\mathcal{S}^{*}$ computes a key pair $\left(s k_{\mathrm{BS}}, p k_{\mathrm{BS}}\right) \leftarrow \mathrm{KG}_{\mathrm{BS}}\left(1^{n}\right)$, it sets $m_{\max }^{*}=10^{n-1}$ and picks two messages $m_{0}=0^{n}, m_{1}=1^{n}$ such that $m_{0} \leq m_{\max }^{*}<m_{1}$. It outputs a public key $p k_{\overline{\mathrm{SF}}}=\left(p k_{\mathrm{BS}}, m_{\max }^{*}\right)$ together with the message $m_{0}, m_{1}$ as defined in the first step of the experiment. Next, $\mathcal{S}^{*}$ has black-box access to two honest user instances (as described in experiment $\left.\operatorname{SFBlind}_{\mathcal{S}^{*}}^{\mathrm{BS}}(n)\right)$ where the first algorithm takes as input $\left(p k_{\overline{\mathrm{SF}}}, m_{b}\right)$ and the second user algorithm receives $\left(p k_{\overline{\mathrm{SF}}}, m_{1-b}\right)$. In both executions $\mathcal{S}^{*}$ acts like the honest signer with key $s k_{\overline{\mathrm{SF}}}=s k_{\mathrm{BS}}$. Then $\mathcal{S}^{*}$ is eventually informed which of the executions has failed, i.e., receives left or right (as $\mathcal{S}^{*}$ has access to honest user instances, the case where both executions fail cannot occur by the completeness condition). The adversary $\mathcal{S}^{*}$ returns $b^{*}=1$ if the left instance has failed, otherwise it returns $b^{*}=0$.

It follows straightforwardly that the adversary $\mathcal{S}^{*}$ succeeds in predicting $b$ with probability 1 .

\subsection{Selective-Failure Blindness for Multiple Executions}

The presumably natural way to extend selective-failure blindness to an arbitrary number of executions with user instances would be as follows. The malicious signer chooses $q$ messages as well as a public key $p k_{\mathrm{BS}}$ and interacts with $q$ user 
instances. We denote by $\pi$ be a random permutation over $\{1,2, \ldots, q\}$. The $i$-th user instance is initiated with the message $m_{\pi(i)}$ and the public key $p k_{\mathrm{BS}}$. If at least one of the user instances aborts, then the adversary is given a binary vector $v$ of length $q$ indicating which of the user algorithms aborted. In the case that each execution allows the user to create a valid signature, then the adversary is given all message-signature pairs in non-permuted order.

In the final step the adversary tries to link a message-signature pair to an execution. There are two possible venues to formalize this. The first one, which we believe reflects much better the idea that the adversary should not be able to determine the order of signed messages, is to ask the adversary two output two indices $i_{0}, i_{1}$ such that $\pi\left(i_{0}\right)<\pi\left(i_{1}\right)$. The second version would be to ask the adversary to predict the connection much more explicitly, demanding to output indices $(i, j)$ such that $\pi(i)=j$. Note that for the case of two executions both notions are equivalent.

Here we give the "order-based" definition and show in in the full version that the two definitions are equivalent, assuming the following strengthening: During the signature issuing and in the final processing phase we give the malicious signer access to an oracle Reveal which for input $i$ returns $\pi(i)$ and the user's signature $\sigma_{i}$ if the execution has already finished successfully. This corresponds to the case that some coins in e-cash systems may have been spent meanwhile. Note that the reveal oracle takes as input a state st ${ }^{\text {rev }}$ where each signature is stored. The adversary's final choice $i_{0}, i_{1}$ must not have been disclosed, of course.

Definition 4. A blind signature scheme $\mathrm{BS}=\left(\mathrm{KG}_{\mathrm{BS}},\langle\mathcal{S}, \mathcal{U}\rangle, \mathrm{Vf}_{\mathrm{BS}}\right)$ is called multi-execution selective-failure blind if it is unforgeable (as in Definition Q and the following holds:

Multi-Execution SF-Blindness. For any efficient algorithm $\mathcal{S}^{*}$ (working in modes find, issue, and reveal) the probability that experiment $\operatorname{MSFBlind}_{\mathcal{S}^{*}}^{\mathrm{BS}}(n)$ returns 1 is negligibly close to $\frac{1}{2}$, where

Experiment MSFBlind ${ }_{\mathcal{S}^{*}}^{\mathrm{BS}}(n)$

$\left(p k_{\mathrm{BS}}, M, \beta_{\text {find }}\right) \leftarrow \mathcal{S}^{*}\left(\right.$ find, $\left.1^{n}\right)$ where $M=\left(m_{1}, \ldots, m_{q}\right)$ with $m_{i} \in\{0,1\}^{n}$ Select a random permutation $\pi$ over $\{1,2, \ldots, q\}$

$\beta_{\text {issue }} \leftarrow \mathcal{S}^{*\left\langle\cdot, \mathcal{U}\left(p k_{\mathrm{BS}}, m_{\pi(1)}\right)\right\rangle^{1}, \ldots,\left\langle\cdot, \mathcal{U}\left(p k_{\mathrm{BS}}, m_{\pi(q)}\right)\right\rangle^{1}, \text { Reveal }\left(\cdot, \pi, s t^{\text {rev }}\right)}$ (issue, $\left.\beta_{\text {find }}\right)$

and let $\sigma_{\pi(1)}, \ldots, \sigma_{\pi(q)}$ denote the (possibly undefined) local outputs

of $\mathcal{U}\left(p k_{\mathrm{BS}}, m_{\pi(1)}\right), \ldots, \mathcal{U}\left(p k_{\mathrm{BS}}, m_{\pi(q)}\right)$, immediately stored in st ${ }^{\text {rev }}$

once an execution finishes (st ${ }^{\text {rev }}$ is initially set to $(\perp, \ldots, \perp)$ );

Reveal $\left(\cdot, \pi, s^{\text {rev }}\right)$ is an oracle, which on input $i$ returns $\left(\pi(i)\right.$, st $\left._{i}^{\text {rev }}\right)$.

Return to $\mathcal{S}^{*}$ all signatures $v=\left(\sigma_{1}, \ldots, \sigma_{q}\right)$ iff all executions

have yielded valid signatures; otherwise return a vector $v \in\{0,1\}^{q}$

where the $i$-th entry is 1 if the $i$-th signature is valid, and 0 otherwise.

$\left(i_{0}, i_{1}\right) \leftarrow \mathcal{S}^{*}$, Reveal $\left(\cdot, \pi, s t^{\text {ev }}\right)\left(\right.$ reveal, $\left.v, \beta_{\text {issue }}\right)$

Return 1 iff $\pi\left(i_{0}\right)<\pi\left(i_{1}\right)$ and $\mathcal{S}^{*}$ has never queried Reveal about $i_{0}, i_{1}$.

The definition of multi-execution selective-failure blindness for the case $q=2$ covers the standard definition of blindness. An adversary $\mathcal{A}$ breaking blindness 
can be used to build an adversary $\mathcal{S}^{*}$ breaking multi-execution selective-failure blindness as follows. The malicious signer $\mathcal{S}^{*}$ executes $\mathcal{A}$ in a black-box way and follows the blindness experiment until $\mathcal{S}^{*}$ receives either the signatures $\sigma_{0}, \sigma_{1}$ or the vector $v$. In case these two valid signatures are given to $\mathcal{S}^{*}$, it forwards both pairs to $\mathcal{A}$ and otherwise it outputs $\perp$. Finally, $\mathcal{S}^{*}$ outputs the decision bit $b^{\prime}$ returned by $\mathcal{A}$. The definition of selective-failure blindness is (semantically) identical to the definition of multi-execution selective failure blindness for the case $q=2$.

Proposition 3. A blind signature scheme which is selective-failure blind, is also multi-execution selective-failure blind.

The proof appears in the full version. The idea is that one is able to guess the two challenge values $i_{0}, i_{1}$ chosen by $\mathcal{S}^{*}$ with sufficiently high probability in advance and one can thus reduce it to the two-execution case.

\subsection{Relation to A-Posteriori Blindness}

In the following we discuss the relation between selective-failure blindness and aposteriori blindness 15. Roughly speaking, a-posteriori blindness advocates that blindness only needs to hold for non-aborted sessions. Hazay et al. formalize this basic idea in an experiment where the adversary first outputs a public key $p k$ together with a message distribution $\mathcal{M}$. The malicious signer then concurrently interacts with $\ell$ honest user instances, where each user instance gets as input the public key $p k$ and a message sampled according to $\mathcal{M}$. Afterwards, when the signer has finished all $\ell$ interactions, it receives $\ell^{\prime}$ message-signature pairs in a randomly permuted order, where $1 \leq \ell^{\prime} \leq \ell$ denotes the number of nonaborted executions. The adversary wins the game if it associates one non-aborted execution to a messages-signature pair. A detailed discussion about a-posteriori blindness in the concurrent setting is given in [15.

From a syntactically point of view there are numerous differences between the definition of selective-failure blindness and a-posteriori blindness. Firstly, the adversary in our security definition picks the messages, whereas in the experiment of a-posteriori blindness it only chooses a message distribution. Secondly, in contrast to a-posteriori blindness, the malicious signer in our case receives the information which of the user instances have aborted. In an e-cash scenario, this corresponds to the case where a user (who may have completed all rounds of the protocol) could not derive a valid coin and informs the signing bank about this problem. Thirdly, we propose two different notions of multi-execution selectivefailure blindness. The first definition (Definition 4) is an ordering-based definition where the adversary has to distinguish the order of two different executions. The second definition (see the full version) is a prediction-based definition where the malicious signer has to link an execution to a message-signature pair.

Finally, the attacker in our definitions has access to a reveal oracle that discloses the message used during a specific execution. Such an oracle is also not considered in the definition of a-posteriori blindness. In the real world, this oracle represents side information the signer obtains, e.g., customer A opens up 
a bank account before customer B. Then customer B cannot withdraw coins before having opened up an account and every meanwhile spent coin has to be from customer A. Note that these side information provide the malicious signer also with information about the non-aborted executions. From a technical point of view, the reveal oracle allows us to prove the equivalence between selective-failure blindness for two executions and for multiple executions, as well as the equivalence of the two types of multi-execution selective-failure blindness definitions.

A natural question is whether the definition of a-posteriori blindness and the definition of multi-execution selective-failure blindness are equivalent for the special case of two executions. To answer this question we briefly recall the counter example of Hazay el al. which shows that a-posteriori blindness does not imply regular blindness. This example consists of a scheme that satisfies a-posteriori blindness but that easily violates blindness. In this scheme, the honest user algorithms validates the first received message from the signer. In the case that this message is improper, then it sends the message $m$ to the signer and aborts afterwards. Since a-posteriori blindness only guarantees blindness for non-aborted sessions, this scheme remains a-posteriori blind. However, it follows easily that this scheme is not blind. Hence, a-posteriori blindness cannot be equivalent to selective-failure blindness, because selective-failure blindness does imply regular blindness.

\section{From Blindness to Selective-Failure Blindness}

In this section we show how to turn every secure blind signature scheme BS into a selective-failure blind signature scheme $\mathrm{BS}_{\mathrm{SF}}$. The high-level idea is to modify BS slightly into $\mathrm{BS}_{\mathrm{SF}}$ by executing $\mathrm{BS}$ with a non-interactive commitment com of the message $m$ (instead of the message itself).

Definition 5 (Commitment Scheme). A (non-interactive) commitment scheme consists of a tuple of efficient algorithms $\mathcal{C}=\left(\mathrm{KG}_{\mathrm{com}}, \mathrm{Com}, \mathrm{Vf}_{\mathrm{com}}\right)$ where

Key Generation. Algorithm $\mathrm{KG}_{\mathrm{com}}\left(1^{n}\right)$ on input the security parameter outputs a key $p k_{\text {com }}$.

Commitment Phase. Algorithm Com takes as input $p k_{\text {com }}$ as well as $m \in$ $\{0,1\}^{n}$ and outputs (decom, com $) \leftarrow \operatorname{Com}\left(p k_{\text {com }}, m\right)$.

Verification. $\mathrm{Vf}_{\mathrm{com}}\left(p k_{\mathrm{com}}, m\right.$, decom, com $)$ outputs a bit.

It is assumed that the commitment scheme is complete, i.e., for any $n \in \mathbb{N}$, any $p k_{\mathrm{com}} \leftarrow \mathrm{KG}_{\mathrm{com}}\left(1^{n}\right)$, for any message $m \in\{0,1\}^{n}$ and any (decom, com) $\leftarrow$ $\operatorname{Com}\left(p k_{\mathrm{com}}, m\right)$ we have $\mathrm{Vf}_{\mathrm{com}}\left(p k_{\mathrm{com}}, m\right.$, decom, com $)=1$.

Security of commitment schemes is defined by secrecy and unambiguity. Secrecy guarantees that the receiver cannot learn the message from the commitment and unambiguity says that the sender cannot change the message anymore once the commitment phase is over. Here we use a slightly different way to define secrecy compared to the literature, but it is easy to see by a hybrid argument that our definition is equivalent: 
Definition 6 (Secure Commitment). $A$ (non-interactive) commitment scheme $\mathcal{C}=\left(\mathrm{KG}_{\text {com }}, \mathrm{Com}, \mathrm{Vf}_{\mathrm{com}}\right)$ is called secure if the following holds:

Secrecy. For any efficient algorithm $\mathrm{R}_{\text {real }}^{*}$ (working in modes find and guess) the probability that experiment $\operatorname{Secrecy}_{\mathrm{R}_{\mathrm{real}}^{*}}^{\mathcal{C}}(n)$ evaluates to 1 is negligibly close to $1 / 2$.

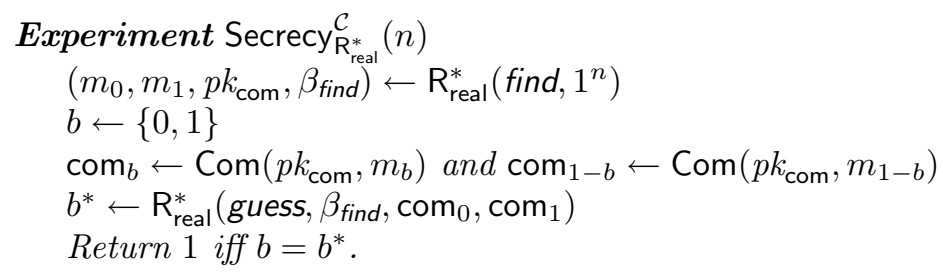

Unambiguity. For any efficient algorithm $\mathrm{S}_{\text {real }}^{*}$ the probability that experiment Unambiguity ${ }_{S_{\text {real }}^{*}}^{\mathcal{C}}(n)$ evaluates to 1 is negligible.

Experiment Unambiguity ${ }_{S_{\text {real }}^{*}}^{\mathcal{C}}(n)$

$$
\begin{aligned}
& p k_{\text {com }} \leftarrow \mathrm{KG}_{\mathrm{com}}\left(1^{n}\right) \\
& \left(m, m^{\prime}, \operatorname{decom}, \operatorname{decom}{ }^{\prime}\right) \leftarrow \mathcal{S}^{*}\left(p k_{\text {com }}\right) \\
& \text { Return } 1 \text { iff } \\
& \quad \mathrm{Vf}_{\mathrm{com}}\left(p k_{\text {com }}, m, \operatorname{decom}, \text { Com }\right)=1 \text { and } \\
& \quad \mathrm{Vf}_{\mathrm{com}}\left(p k_{\mathrm{com}}, m^{\prime}, \operatorname{decom}^{\prime}, \text { Com }\right)=1 \text { as well as } m \neq m^{\prime} .
\end{aligned}
$$

Note that such commitment schemes exist under standard assumptions like pseudorandom generators [19] or hash functions 8. In order to use a commitment in a blind signature scheme - which we defined to take messages of $n$ bits - we need that the commitment scheme is length-invariant, meaning that for $n$-bit messages the commitment itself is also $n$ bits. This can always be achieved by using a collision-resistant hash function (with $n$ bits output) on top.

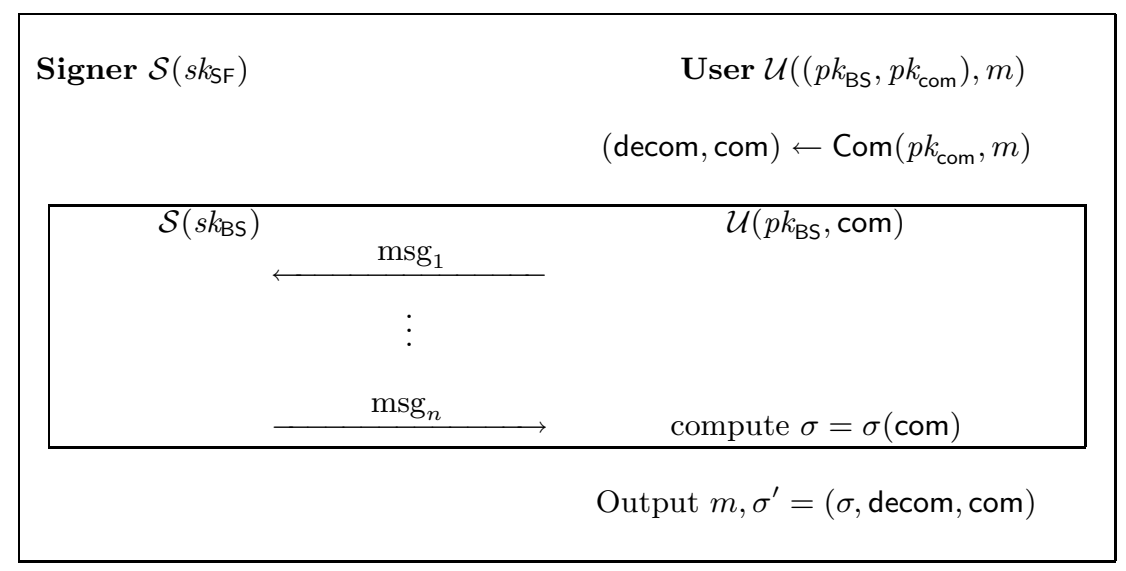

Fig. 1. Issue protocol of the blind signature scheme $\mathrm{BS}_{\mathrm{SF}}$ 
Construction 1 (Selective-Failure Blind Signature Scheme $\mathrm{BS}_{\mathrm{SF}}$ ). Let $\mathrm{BS}=\left(\mathrm{KG}_{\mathrm{BS}},\langle\mathcal{S}, \mathcal{U}\rangle, \mathrm{Vf}_{\mathrm{BS}}\right)$ be a blind signature scheme and let $\mathcal{C}$ be a lengthinvariant commitment scheme. Define the blind signature scheme $\mathrm{BS}_{\mathrm{SF}}$ through the following three procedures:

Key Generation. The generation algorithm $\mathrm{KG}_{\mathrm{SF}}\left(1^{n}\right)$ executes the key generation algorithm of the blind signature scheme $\mathrm{BS},\left(s k_{\mathrm{BS}}, p k_{\mathrm{BS}}\right) \leftarrow \mathrm{KG}_{\mathrm{BS}}\left(1^{n}\right)$. It also runs the key generation algorithm for the commitment scheme, $p k_{\mathrm{com}} \leftarrow$ $\mathrm{KG}_{\mathrm{com}}\left(1^{n}\right)$. It returns the private key $s k_{\mathrm{SF}}=s k_{\mathrm{BS}}$ and the public key $p k_{\mathrm{SF}}=$ $\left(p k_{\mathrm{BS}}, p k_{\mathrm{com}}\right)$.

Signature Issue Protocol. The interactive signature issue protocol for message $m \in\{0,1\}^{n}$ is described in Figure 1 .

Signature Verification. The verification algorithm $\mathrm{Vf}_{\mathrm{SF}}\left(p k_{\mathrm{SF}}, m, \sigma^{\prime}\right)$ for $\sigma^{\prime}=$ $(\sigma$, decom, com $)$ returns 1 iff $\mathrm{Vf}_{\mathrm{BS}}\left(p k_{\mathrm{BS}}, \sigma, \mathrm{com}\right)=1$ and $\mathrm{Vf}_{\mathrm{com}}\left(p k_{\mathrm{com}}, m\right.$, decom, com $)=1$.

Theorem 1. If $\mathrm{BS}$ is a secure blind signature scheme and $\mathcal{C}$ is a secure, lengthinvariant commitment scheme, then the scheme $\mathrm{BS}_{\mathrm{SF}}$ in Construction 1 is a selective-failure blind signature scheme.

We note that, if the starting blind signature scheme provides statistical blindness, and the commitment scheme is also statistically-hiding, then the derived protocol achieves selective-failure blindness in a statistical sense. This can be seen from the proof of the theorem, which is split into two claims, covering unforgeability and selective-failure blindness:

CLAIM 1: BS $\mathrm{SF}$ is unforgeable.

In the proof we distinguish between two cases. The first case occurs if the adversary $\mathcal{U}^{*}$ succeeds in outputting $k+1$ valid pairs $m_{i}, \sigma_{i}^{\prime}=\left(\sigma_{i}, \operatorname{decom}_{i}, \operatorname{com}_{i}\right)$ such that the commitments $\operatorname{com}_{i}$ are pairwise different. But then we can break the unforgeability of the underlying blind signature scheme BS. In the second case $\mathcal{U}^{*}$ succeeds and at least two commitments $\operatorname{com}_{i}, \operatorname{com}_{j}($ with $i \neq j)$ are identical. But then we can break the unambiguity of the commitment scheme $\mathcal{C}$.

Proof. Assume to the contrary that the resulting selective-failure blind signature scheme $\mathrm{BS}_{\mathrm{SF}}$ is not unforgeable. Then there exists an adversary $\mathcal{U}^{*}$ breaking unforgeability with noticeable probability, i.e., on input $p k_{\mathrm{SF}}$ the algorithm $\mathcal{U}^{*}$ returns $k+1$ valid signatures $\sigma_{i}^{\prime}=\left(\sigma_{i}\right.$, decom $\left._{i}, \operatorname{com}_{i}\right)$ for messages $m_{i}$ after at most $k$ interactions with the honest signer $\mathcal{S}$. Note that here we do not deal with user aborts and count any initiated interaction; the case of counting only completed interactions is taken care of in the next section.

We first take a look at the success probability of $\mathcal{U}^{*}$, we have

$$
\psi(n):=\operatorname{Prob}\left[\operatorname{Forge}_{\mathcal{U}^{*}}^{\mathrm{BS}}(n)=1\right]
$$

where $\psi(n)$ is noticeable. This probability can be separated according to the two exclusive events that $\mathcal{U}^{*}$ succeeds and all commitments $\operatorname{com}_{i}$ are different, with 
the corresponding probability denoted by $\psi_{0}(n)$, and into the case where $\mathcal{A}_{\mathrm{SF}}$ succeeds and at least two commitments are identical (with probability $\psi_{1}(n)$ ) According to our assumption that $\psi(n)$ is noticeable, $\psi_{0}(n)$ or $\psi_{1}(n)$ (or both) must be noticeable.

We next construct out of $\mathcal{U}^{*}$ algorithms $\mathcal{A}_{\text {UNF }}$ and $\mathcal{A}_{\text {UNA }}$ against unforgeability of BS and unambiguity of the commitment scheme $\mathcal{C}$.

Attacking Unforgeability. The adversary $\mathcal{A}_{\mathrm{UNF}}$ takes as input the public key $p k_{\mathrm{BS}}$ of the blind signature scheme BS and works as follows. It executes the key generation algorithm of the commitment scheme $p k_{\text {com }} \leftarrow \mathrm{KG}_{\text {com }}\left(1^{n}\right)$ and runs a black-box simulation of $\mathcal{U}^{*}$ on input $p k_{\mathrm{SF}}=\left(p k_{\mathrm{BS}}, p k_{\text {com }}\right)$. The signer instances in the attack of $\mathcal{U}^{*}$ are simulated with the help of the external signer instances accessible by $\mathcal{A}_{\mathrm{UNF}}$, i.e., adversary $\mathcal{A}_{\mathrm{UNF}}$ relays the communication between $\mathcal{U}^{*}$ and its signer instance oracle $\mathcal{S}\left(s k_{\mathrm{BS}}\right.$ ) (as described in experiment Forge $\mathcal{U}^{\mathrm{BS}}$ ). When $\mathcal{U}^{*}$ finishes its attack, it outputs $k+1$ message-signatures pairs $m_{i}, \sigma_{i}^{\prime}$ after at most $k$ interactions. Now $\mathcal{A}_{\text {UNF }}$ parses each $\sigma_{i}^{\prime}$ as $\left(\sigma_{i}, \operatorname{decom}_{i}, \operatorname{com}_{i}\right)$ and returns the $k+1$ pairs $\operatorname{com}_{i}, \sigma_{i}$ and stops.

Assume that $\psi_{0}(n)$, the probability that $\mathcal{U}^{*}$ succeeds and all $\mathrm{com}_{i}$ 's are distinct, is noticeable. Then, since the simulation is perfect from the viewpoint of $\mathcal{U}^{*}$, adversary $\mathcal{A}_{\text {UNF }}$ succeeds in outputting $k+1$ valid pairs $\operatorname{com}_{i}, \sigma_{i}$ for distinct "messages" com $_{i}$ with noticeable probability, too, contradicting the unforgeability property of the underlying blind signature scheme. Note also that the numbers of initiated and completed executions are identical in both cases.

Attacking Unambiguity. In order to break the unambiguity of $\mathcal{C}$, the adversary $\mathcal{A}_{\text {UNA }}$ takes as input the public key $p k_{\text {com }}$ of the commitment scheme $\mathcal{C}$ and works as follows. It executes the key generation algorithm of the blind signature scheme $\left(s k_{\mathrm{BS}}, p k_{\mathrm{BS}}\right) \leftarrow \mathrm{KG}_{\mathrm{BS}}\left(1^{n}\right)$ as well as a the honest signer algorithms $\mathcal{S}\left(s k_{\mathrm{BS}}\right)$ and runs a black-box simulation of $\mathcal{U}^{*}$ on input $p k_{\mathrm{SF}}=\left(p k_{\mathrm{BS}}, p k_{\text {com }}\right)$. Note that running the program of the honest signer on input $s k_{\mathrm{BS}}$ simulates each execution with a signer instance. Algorithm $\mathcal{U}^{*}$ eventually returns $k+1$ message-signature pairs $\left(m_{i}, \sigma_{i}^{\prime}\right)$ after at most $k$ interactions with $\mathcal{S}$. The adversary $\mathcal{A}_{\text {UNA }}$ then checks if there are valid signatures with $\operatorname{com}_{i}=\mathrm{com}_{j}$ for some $i \neq j$ and, if so, outputs two tuples $\left(m_{i}, \operatorname{decom}_{i}, \operatorname{com}_{i}\right),\left(m_{j}\right.$, decom $\left._{j}, \operatorname{com}_{j}\right)$ such that $m_{i} \neq m_{j}$ and $\operatorname{com}_{i}=\operatorname{com}_{j}$. If not, it outputs a failure message.

For the analysis note that the simulation again perfectly mimics the original attack of $\mathcal{U}^{*}$. Hence, if $\psi_{1}(n)$ is noticeable, then such $\operatorname{com}_{i}=\operatorname{com}_{j}$ with valid decommitments for $m_{i} \neq m_{j}$ appear with noticeable probability, and the commitment adversary $\mathcal{A}_{\text {UNA }}$ therefore finds an ambiguous commitment with this probability, too. But this clearly violates the security of the commitment scheme $\mathcal{C}$.

Claim 2: BS $\mathrm{SF}$ is selective-failure blind.

The high-level idea of the proof is as follows. We again distinguish between two cases. In the first case the adversary $\mathcal{A}_{\mathrm{SF}}$ succeeds with noticeable probability and both message-signature pairs are valid. But then we show how to break the blindness property of the underlying blind signature scheme BS. We next argue 
that in the case where $\mathcal{A}_{\mathrm{SF}}$ succeeds with noticeable probability and forces at least one of the user algorithms to fail, then we are able to break the secrecy of the commitment scheme (because then the only information available to the signer are the commitments of the messages).

Proof. Assume towards contradiction that the resulting blind signature scheme $\mathrm{BS}_{\mathrm{SF}}$ is not selective-failure blind, and that there exists a successful adversary $\mathcal{A}_{\mathrm{SF}}$ against selective-failure blindness. Let

$$
\delta(n):=\operatorname{Prob}\left[\operatorname{SFBlind}_{\mathcal{A}_{\mathrm{SF}}}^{\mathrm{BS}}(n)=1\right]=\frac{1}{2}+\epsilon(n)
$$

where $\epsilon(n)=\delta(n)-\frac{1}{2}$ is noticeable. We divide the success case according to the two exclusive events that $\mathcal{A}_{\mathrm{SF}}$ succeeds and that both message-signature pairs are valid (event valid) and into the case where $\mathcal{A}_{\mathrm{SF}}$ succeeds and at least one of the signatures is not valid (event $\neg$ valid). Then,

$$
\begin{aligned}
& \operatorname{Prob}\left[\operatorname{SFBlind}_{\mathcal{A}_{\mathrm{SF}}}^{\mathrm{BS}}(n)=1\right]-\frac{1}{2} \\
&=\operatorname{Prob}[\text { valid }] \cdot\left(\operatorname{Prob}\left[\operatorname{SFBlind}_{\mathcal{A}_{\mathrm{SF}}}^{\mathrm{BS}}(n)=1 \mid \text { valid }\right]-\frac{1}{2}\right) \\
& \quad+\operatorname{Prob}[\neg \text { valid }] \cdot\left(\operatorname{Prob}\left[\operatorname{SFBlind}_{\mathcal{A}_{\mathrm{SF}}}^{\mathrm{BS}}(n)=1 \mid \neg \text { valid }\right]-\frac{1}{2}\right) .
\end{aligned}
$$

According to our assumption that $\delta(n)$ is noticeable, either the first term, denoted $\delta_{0}(n)$, or the second term $\delta_{1}(n)$ has to be noticeable (or both are noticeable). We next turn $\mathcal{A}_{\mathrm{SF}}$ into algorithms $\mathcal{A}_{\text {blind }}$ and $\mathcal{A}_{\text {com }}$ against regular blindness and secrecy of the commitment scheme, respectively.

Attacking Blindness. The adversary $\mathcal{A}_{\text {blind }}$ works as follows. It runs a blackbox simulation of $\mathcal{A}_{\mathrm{SF}}$, which initially outputs two messages $\left(m_{0}, m_{1}\right)$ together with a public key $p k_{\mathrm{SF}}$. The adversary $\mathcal{A}_{\text {blind }}$ extracts $p k_{\mathrm{BS}}$ and $p k_{\text {com }}$ from $p k_{\mathrm{SF}}$ and calculates the commitments (and decommitments) (decom ${ }_{0}$, com $\left._{0}\right) \leftarrow$ $\operatorname{Com}\left(p k_{\text {com }}, m_{0}\right)$ and $\left(\operatorname{decom}_{1}, \operatorname{com}_{1}\right) \leftarrow \operatorname{Com}\left(p k_{\text {com }}, m_{1}\right)$. It outputs $\operatorname{com}_{0}, \operatorname{com}_{1}$ and $p k_{\mathrm{BS}}$. It is given access to user instances $\mathcal{U}\left(p k_{\mathrm{BS}}, \operatorname{com}_{b}\right)$ and $\mathcal{U}\left(p k_{\mathrm{BS}}, \operatorname{com}_{1-b}\right)$ for a unknown bit $b$ and relays the communication between these instances and $\mathcal{A}_{\mathrm{SF}}$. If, at the end, at least one of the (external) user algorithms fails, then $\mathcal{A}_{\text {blind }}$ outputs a random bit and stops. Otherwise, it augments $\sigma_{0}, \sigma_{1}$ to $\sigma_{0}^{\prime}=\left(\sigma_{0}\right.$, decom $_{0}$, com $\left._{0}\right)$ and $\sigma_{1}^{\prime}=\left(\sigma_{1}\right.$, decom $_{1}$, com $\left._{1}\right)$ and returns the two signatures $\sigma_{0}^{\prime}, \sigma_{1}^{\prime}$ (obtained by the external user algorithms) to $\mathcal{A}_{\mathrm{SF}}$. The final output of $\mathcal{A}_{\text {blind }}$ consists of the bit $b^{*}$ returned by $\mathcal{A}_{\mathrm{SF}}$.

Note that $\mathcal{A}_{\text {blind }}$ simulates the experiment $\operatorname{SFBlind}_{\mathcal{A}_{\mathrm{SF}}}^{\mathrm{BS}}(n)$ by executing the blindness experiment for the underlying blind signature scheme BS and by computing the commitments internally. Hence, the case where both messagesignature pairs are valid is the one where experiment $\operatorname{SFBlind}_{\mathcal{A}_{\mathrm{SF}}}^{\mathrm{BS}}(n)$ is identical to experiment Blind ${ }_{\mathcal{A}_{\text {blind }}}^{\mathrm{BS}}(n)$. If one of the signatures is invalid, then $\mathcal{A}_{\text {blind }}$ returns a random bit. Therefore, the success probability of $\mathcal{A}_{\text {blind }}$ in experiment Blind $_{\mathcal{A}_{\text {blind }}}^{\mathrm{BS}}(n)$ can be calculated as: 


$$
\begin{aligned}
& \operatorname{Prob}\left[\operatorname{Blind}_{\mathcal{A}_{\text {blind }}}^{\mathrm{BS}}(n)=1\right] \\
& =\operatorname{Prob}\left[b=b^{*} \wedge \neg \text { valid }\right]+\operatorname{Prob}\left[b=b^{*} \wedge \text { valid }\right] \\
& =\operatorname{Prob}\left[b=b^{*} \mid \text { valid }\right] \cdot \operatorname{Prob}[\text { valid }]+\operatorname{Prob}\left[b=b^{*} \mid \neg \text { valid }\right] \cdot \operatorname{Prob}[\neg \text { valid }] \text {. } \\
& =\operatorname{Prob}[\text { valid }] \cdot \operatorname{Prob}\left[b=b^{*} \mid \text { valid }\right]+\frac{1}{2} \cdot(1-\operatorname{Prob}[\text { valid }]) \\
& =\operatorname{Prob}[\text { valid }] \cdot \operatorname{Prob}\left[\operatorname{SFBlind}_{\mathcal{A}_{\mathrm{SF}}}^{\mathrm{BS}}(n)=1 \mid \text { valid }\right]+\frac{1}{2} \cdot(1-\operatorname{Prob}[\text { valid }]) \\
& =\frac{1}{2}+\operatorname{Prob}[\text { valid }] \cdot\left(\operatorname{Prob}\left[\operatorname{SFBlind}_{\mathcal{A}_{\mathrm{SF}}}^{\mathrm{BS}}(n)=1 \mid \text { valid }\right]-\frac{1}{2}\right) \\
& =\frac{1}{2}+\delta_{0}(n) \text {. }
\end{aligned}
$$

According to our assumption that $\delta_{0}(n)$ is noticeable it follows that $\mathcal{A}_{\text {blind }}$ breaks the blindness of the underlying blind signature scheme BS with noticeable probability. This, however, contradicts our assumption that BS is a secure blind signature scheme.

Attacking Secrecy of the Commitment. In order to break the secrecy of the commitment scheme $\mathcal{C}$, the adversary $\mathcal{A}_{\text {com }}$ executes a black-box simulation of $\mathcal{A}_{\mathrm{SF}}$, which initially outputs two messages $\left(m_{0}, m_{1}\right)$ as well as a public key $p k_{\mathrm{SF}}$. The adversary $\mathcal{A}_{\text {com }}$ extracts the keys $p k_{\text {com }}$ and $p k_{\mathrm{BS}}$ from $p k_{\mathrm{SF}}$ and outputs $\left(m_{0}, m_{1}, p k_{\text {com }}\right)$ for the secrecy experiment of the commitment scheme. It then receives two commitments $\operatorname{com}_{0}, \mathrm{com}_{1}$, one for message $m_{b}$ and the other one for message $m_{1-b}$ (without knowing which commitment corresponds to which message).

The adversary now runs (in the role of the honest user $\mathcal{U}\left(p k_{\mathrm{BS}}, \mathrm{com}_{0}\right)$ and $\left.\mathcal{U}\left(p k_{\mathrm{BS}}, \mathrm{com}_{1}\right)\right)$ the selective-failure blindness experiment with $\mathcal{A}_{\mathrm{SF}}$. At the end of the issue protocol each user instance returns either a signature for the commitment or $\perp$. In the case that both user algorithms return a valid signature, then $\mathcal{A}_{\text {com }}$ outputs a random bit $b^{*}$ and stops. Otherwise, if both user algorithms have failed, then $\mathcal{A}_{\text {com }}$ sends the value both to $\mathcal{A}_{\mathrm{SF}}$. In the case that the first user algorithm has failed, then $\mathcal{A}_{\text {com }}$ returns left to $\mathcal{A}_{\mathrm{SF}}$ and else (if the second user algorithm has failed), it forwards right to $\mathcal{A}_{\mathrm{SF}}$. The final output of $\mathcal{A}_{\text {com }}$ consists of the bit $b^{*}$ returned by $\mathcal{A}_{\mathrm{SF}}$.

The adversary $\mathcal{A}_{\text {com }}$ simulates the experiment of selective-failure blindness perfectly, up to the point where it obtains the (possibly undefined) signatures. Given that at least one of them is invalid, the simulation corresponds to the case SFBlind ${ }_{\mathcal{A}_{\mathrm{SF}}}^{\mathrm{BS}}(n)$ (given $\neg$ valid) for the same choice $b$ as in the commitment experiment. Else, $\mathcal{A}_{\text {com }}$ outputs a random bit. A simple calculation similar to the previous case now shows that

$$
\operatorname{Prob}\left[\operatorname{Secrecy}_{\mathrm{R}_{\text {real }}^{*}}^{\mathcal{C}}(n)=1\right]=\frac{1}{2}+\delta_{1}(n)
$$

If $\delta_{1}(n)$ is noticeable, it follows that $\mathcal{A}_{\text {com }}$ breaks the secrecy of the commitment scheme with noticeable probability, contradicting the security of $\mathcal{C}$. 


\section{$5 \quad$ Unforgeability and User Aborts}

In this section we consider executions in which an adversarial controlled user may abort sessions and the unforgeability requirement with respect to initiated or completed executions with the signer. For sake of distinction we call the requirement where the adversary has to find $k+1$ valid message-signature pairs after $k$ initiated executions weak unforgeability, and the originally given definition charging only completed executions unforgeability under user aborts.

We show in the following that every three-move blind signature scheme, which is weakly unforgeable is also unforgeable under user aborts. Note that in threemove schemes, for a meaningful protocol, the first message is always sent by the signer. As such we may think of two-move schemes as having an additional first move in which the signer simply sends an empty message (although the claim for two-move schemes follows straightforwardly anyway).

We remark that we leave the scheduling of transmissions fully up to the adversary controlling the users, i.e., the adversary decides when to send messages to the signer and when the signer's messages are delivered to the user. Only the signer's output ok is given immediately after the signer's final message has been delivered.

Theorem 2. Every secure blind signature scheme with at most three moves is unforgeable under user aborts.

The proof idea is that we can delay the delivery of the user's message in an execution till we can be sure that the adversary completes this execution. If the execution is not completed, then we can simply disregard the original user message, finish the protocol ourselves as an honest user and create another valid signature in addition to the forgeries of the adversary. The full proof appears in the full version.

We note that the result above is optimal in the sense that for four or more moves no such claim can be made (if there are secure schemes with two moves):

Proposition 4. Every secure blind signature scheme BS with two moves can be converted into a secure blind signature scheme $\mathrm{BS}_{\overline{\mathrm{UuA}}}$ with four moves, which is weakly unforgeable but not unforgeable under user aborts.

The claim follows by adding two dummy messages at the end, one from the user to signer and one from the signer to the user, such that a malicious user can abort before these dummy messages are exchanged and is still able to derive a signature. The proof appears in the full version.

The previous proposition does not rule out that there is a transformation turning schemes with four or more moves into unforgeable ones under user aborts. An apparent approach is to ignore the original protocol and two run a scheme, which already has this property (like Chaum's two-move blind signature scheme in the random oracle model). Yet, it is preferable of course to have a lightweight transformation adhering to the basics of the underlying protocol (like the avoidance of random oracles or general but expensive multi-party protocols). 


\section{Selective Failures and Adaptive Oblivious Transfer}

Camenisch et al. 7] also show how to construct an adaptive oblivious transfer protocol out of any unique selective-failure blind signature scheme (in the random oracle model). Roughly speaking, uniqueness means that each message has only one signature per public key. More formally, a blind signature scheme is unique 12,7] if for every (possibly maliciously chosen) public key $p k_{\mathrm{BS}}$ and every message $m \in\{0,1\}^{*}$, there exists at most one signature $s \in\{0,1\}^{*}$ such that $\mathrm{Vf}_{\mathrm{BS}}\left(p k_{\mathrm{BS}}, m, s\right)=1$.

In this section we focus on the question whether our transformation turning every blind signature into one with selective-failure blindness is applicable. We have already mentioned in the introduction that the initial commitment destroys uniqueness of the blind signature scheme because each message may have several valid signatures per key pair. Here we show that is nonetheless possible to build an adaptive $k$-out-of- $N$ oblivious transfer protocol out of any unique blind signature scheme by applying our transformation. The following construction is a modification of the protocol in 7] and, because of the problems with uniqueness, we have to prove the security of this construction from scratch, digging also into the proof of selective-failure blindness for our transformation.

\subsection{Simulatable Adaptive Oblivious Transfer}

Oblivious Transfer (OT), proposed by Rabin [23], is an interactive protocol between a sender $\mathrm{S}$ and a receiver $\mathrm{R}$. The sender in this protocol gets as input $N$ messages $m_{1}, \ldots, m_{N}$ and the receiver $\mathrm{R}$ wishes to retrieve the message $m_{c}$. OT protocols must satisfy the following two security properties: firstly, the sender $S$ does not find out the receiver's choice $c \in\{1, \ldots, N\}$ and, secondly, the receiver only obtains $m_{c}$ and does not gain any information about the other messages $m_{i}$ for $i \neq c$. For adaptive $k$-out-of- $N$ oblivious transfer, $\mathrm{OT}_{k \times 1}^{N}$, the receiver requests $k$ of these $N$ messages in rounds where the $i$-th choice is based on the previously obtained messages. We refer the reader to [7, 20] for more information.

As in [7] we consider the real-world/ideal-world paradigm for both sender and receiver security (simulatable oblivious transfer). This paradigm compares the execution of an OT protocol in the real-world with an ideal implementation (see for example [4]). In the real-world experiment, both parties jointly execute the interactive protocol, whereas in the ideal-world the functionality is realized through a trusted third party. In our case this means that the sender first hands over the messages to the trusted party, and then the receiver can adaptively obtain messages. To capture failures we let the ideal-world sender in each retrieval also send a bit $b$, indicating whether the transfer should succeed or abort. We note that this bit is independent of the choice of the receiver, reflecting the fact that the abort should not depend on the receiver's input. Due to the limited space, we review the formal security definition in the full version. 


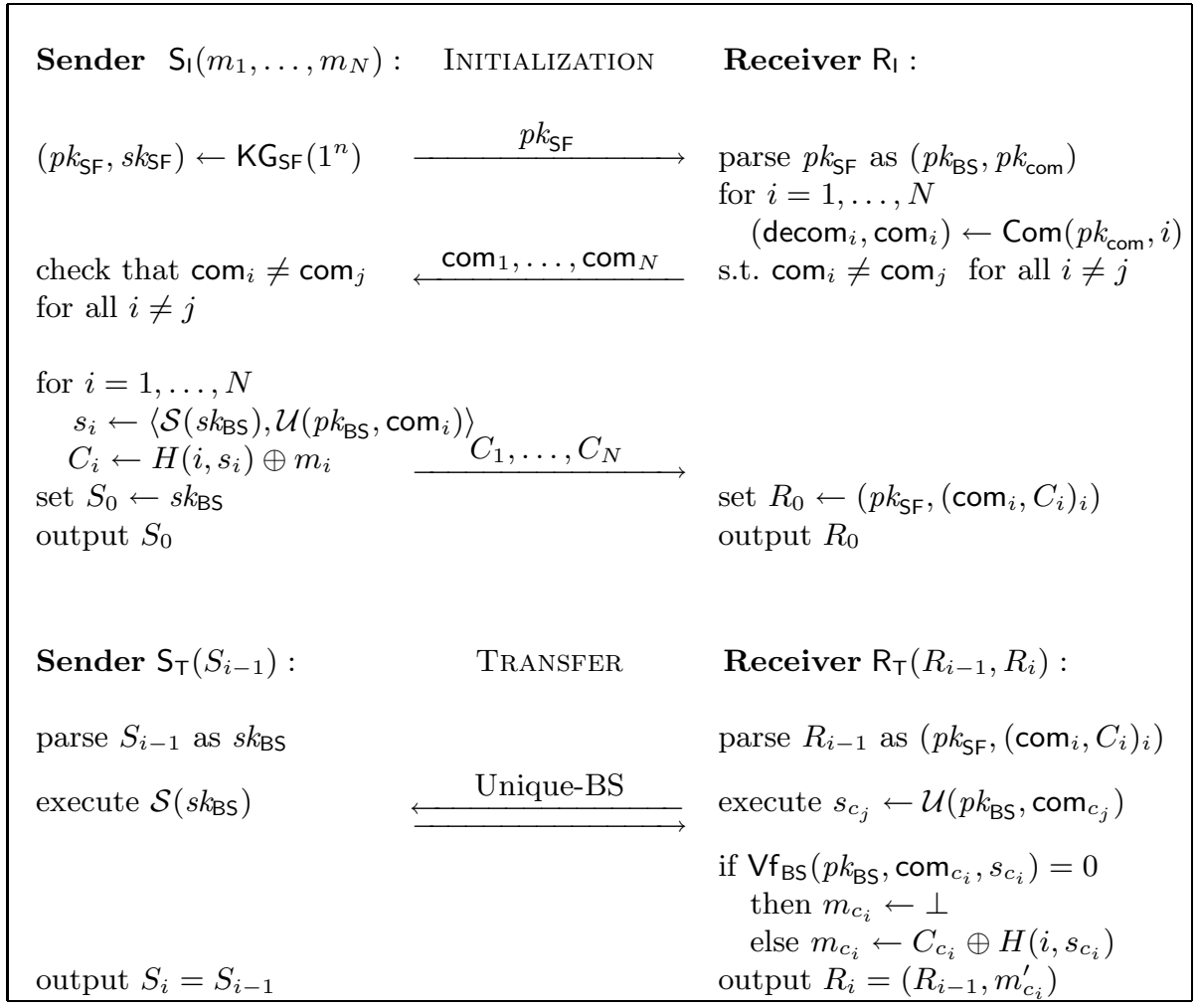

Fig. 2. A $k$-out-of- $N$ oblivious transfer protocol using a random oracle $H$ and any unique blind signature scheme BS

\subsection{Construction}

Our construction, depicted in Figure 2, is a modification of the $\mathrm{OT}_{k \times 1}^{N}$ protocol of Camenisch et al. and consists of a black-box construction using any unique (not necessarily selective-failure) blind signature scheme. The sender in the first step of the protocol generates a key-pair for the blind signature scheme and sends it to the receiver. The receiver, in return, hands $N$ distinct commitments (for values $1,2, \ldots, N$, represented as $n$-bit-strings each) over to the sender. These commitments serve as "messages" for the signature generation. Note that distinctiveness of the commitments holds with high probability by the binding property.

After the sender has verified that all commitments are distinct, it encrypts each message in its database by xoR-ing the message $m_{i}$ with $H\left(i, s_{i}\right)$, where $i$ is the index of the $i$-th commitment $\operatorname{com}_{i}$ and $s_{i}$ is the unique signature of message $\mathrm{com}_{i}$ under $p k_{\mathrm{BS}}$. The sender can easily compute this signature locally by running the signature issue protocol with the help of the signing key and an honest user instance for "message" $\operatorname{com}_{i}$.

After having finished the initialization phase, both parties engage in a transfer phase that consists of a run of the unique blind signature scheme. In the case 
that the receiver wishes to obtain the $i$-th message $m_{i}$, then it has to choose the commitment $\operatorname{com}_{i}$ (as the message to be signed) during the signature issue protocol.

From a high-level point of view unforgeability guarantees that the receiver cannot receive more messages than interactions took place (sender's security) and blindness guarantees that the sender cannot tell which message has been signed (receiver's security).

Theorem 3. If the unique blind signature scheme BS is unforgeable then the $O T_{k \times 1}^{N}$ scheme depicted in Figure 2 is sender-secure in the random oracle model.

The proof of this (and the following) theorem appears in the full version.

Theorem 4. If $\mathrm{BS}$ is a secure blind signature scheme and $\mathcal{C}$ is a secure, lengthinvariant commitment scheme, then the $O T_{k \times 1}^{N}$ scheme depicted in Figure 2 is receiver-secure in the random oracle model.

\section{Acknowledgments}

We thank Heike Busch, Jonathan Katz, and the anonymous reviewers for valuable comments. Both authors are supported by the Emmy Noether Program Fi 940/2-1 of the German Research Foundation (DFG).

\section{References}

1. Abe, M.: A Secure Three-Move Blind Signature Scheme for Polynomially Many Signatures. In: Pfitzmann, B. (ed.) EUROCRYPT 2001. LNCS, vol. 2045, pp. 136151. Springer, Heidelberg (2001)

2. Asokan, N., Shoup, V., Waidner, M.: Optimistic fair exchange of digital signatures. In: Nyberg, K. (ed.) EUROCRYPT 1998. LNCS, vol. 1403, pp. 591-606. Springer, Heidelberg (1998)

3. Boldyreva, A.: Efficient Threshold Signatures, Multisignatures and Blind Signatures Based on the Gap-Diffie-Hellman-Group Signature Scheme. In: Desmedt, Y.G. (ed.) PKC 2003. LNCS, vol. 2567, pp. 31-46. Springer, Heidelberg (2002)

4. Canetti, R.: Security and Composition of Multiparty Cryptographic Protocols. Journal of Cryptology 13, 143-202 (2000)

5. Chaum, D.: Blind Signatures for Untraceable Payments. In: Chaum, D. (ed.) Advances in Cryptology - Crypto 1982, pp. 199-203. Plemum, New York (1983)

6. Camenisch, J.L., Koprowski, M., Warinschi, B.: Efficient blind signatures without random oracles. In: Blundo, C., Cimato, S. (eds.) SCN 2004. LNCS, vol. 3352, pp. 134-148. Springer, Heidelberg (2005)

7. Camenisch, J.L., Neven, G., Shelat, A.: Simulatable adaptive oblivious transfer. In: Naor, M. (ed.) EUROCRYPT 2007. LNCS, vol. 4515, pp. 573-590. Springer, Heidelberg (2007)

8. Damgärd, I., Pedersen, T., Pfitzmann, B.: On the Existence of Statistically Hiding Bit Commitment Schemes and Fail-Stop Signatures. Journal of Cryptology 10(3), 163-194 (1997) 
9. Fischlin, M.: Round-optimal composable blind signatures in the common reference string model. In: Dwork, C. (ed.) CRYPTO 2006. LNCS, vol. 4117, pp. 60-77. Springer, Heidelberg (2006)

10. Fujioka, A., Okamoto, T., Ohta, K.: A Practical Secret Voting Scheme for Large Scale Elections. In: Zheng, Y., Seberry, J. (eds.) AUSCRYPT 1992. LNCS, vol. 718, pp. 244-251. Springer, Heidelberg (1993)

11. Garay, J.A., MacKenzie, P.D., Prabhakaran, M., Yang, K.: Resource fairness and composability of cryptographic protocols. In: Halevi, S., Rabin, T. (eds.) TCC 2006. LNCS, vol. 3876, pp. 404-428. Springer, Heidelberg (2006)

12. Goldwasser, S., Ostrovsky, R.: Invariant signatures and non-interactive zeroknowledge proofs are equivalent. In: Brickell, E.F. (ed.) CRYPTO 1992. LNCS, vol. 740, pp. 228-245. Springer, Heidelberg (1993)

13. Goldreich, O.: The Foundations of Cryptography, vol. 2. Cambridge University Press, Cambridge (2004)

14. Horvitz, O., Katz, J.: Universally-composable two-party computation in two rounds. In: Menezes, A. (ed.) CRYPTO 2007. LNCS, vol. 4622, pp. 111-129. Springer, Heidelberg (2007)

15. Hazay, C., Katz, J., Koo, C.-Y., Lindell, Y.: Concurrently-secure blind signatures without random oracles or setup assumptions. In: Vadhan, S.P. (ed.) TCC 2007. LNCS, vol. 4392, pp. 323-341. Springer, Heidelberg (2007)

16. Juels, A., Luby, M., Ostrovsky, R.: Security of blind digital signatures. In: Kaliski Jr., B.S. (ed.) CRYPTO 1997. LNCS, vol. 1294, pp. 150-164. Springer, Heidelberg (1997)

17. Kiayias, A., Zhou, H.-S.: Two-Round Concurrent Blind Signatures without Random Oracles. Number 2005/435 in Cryptology eprint archive (2005), eprint.iacr.org

18. Kiayias, A., Zhou, H.-S.: Equivocal blind signatures and adaptive UC-security. In: Canetti, R. (ed.) TCC 2008. LNCS, vol. 4948, pp. 340-355. Springer, Heidelberg (2008)

19. Naor, M.: Bit Commitment Using Pseudo-Randomness. Journal of Cryptology 4(2), 151-158 (1991)

20. Naor, M., Pinkas, B.: Computationally Secure Oblivious Transfer. Journal of Cryptology 18(1), 1-35 (2005)

21. Okamoto, T.: Efficient Blind and Partially Blind Signatures Without Random Oracles. In: Halevi, S., Rabin, T. (eds.) TCC 2006. LNCS, vol. 3876, pp. 80-99. Springer, Heidelberg (2006)

22. Pointcheval, D., Stern, J.: Security Arguments for Digital Signatures and Blind Signatures. Journal of Cryptology 13(3), 361-396 (2000)

23. Rabin, M.: How to Exchange Secrets by Oblivious Transfer. Technical Report TR81, Aiken Computation Laboratory (1981) 